El ectric fi el d and thermal diffusivity prof iles of a compact hel i cal system hel i ot ron/t or satron pl asma i $n$ a pl at eau regi re

\begin{tabular}{|l|l|}
\hline $\begin{array}{l}\text { j our nal or } \\
\text { publ i cat } i \text { on } \mathrm{titl} \text { e }\end{array}$ & Physi cs of FI ui ds B \\
\hline vol une & Vol . 4 \\
\hline number & I ssue 5 \\
\hline page range & pp. 1360-1361 \\
\hline year & 1992- 05- 01 \\
\hline URL & ht t p: //hdl . handl e. net /10655/113 \\
\hline
\end{tabular}


The purpose of this Rrief Communications section is to present important research results of more limited scope than regular articles appearing in Physics of Fluids B. Submission of material of a peripheral or cursory nature is strongly discouraged. Brief Communications cannot exceed three printed pages in length, including space allowed for title, figures, tables, references, and an abstract limited to about 100 words.

\title{
Electric field and thermal diffusivity profiles of a compact helical system heliotron/torsatron plasma in a plateau regime
}

\author{
K. Ida, H. Yamada, H. Iguchi, S. Hidekuma, H. Sanuki, K. Yamazaki, and the CHS Group \\ National Institute for Fusion Science, Nagoya, 464-01 Japan \\ (Received 1 July 1991; accepted 28 January 1992) \\ The radial electric field profiles are derived from poloidal rotation velocity for a plateau \\ regime plasma heated by a neutral beam in a CHS device. The measured electric field is more \\ negative than the neoclassical prediction especially near the plasma periphery. However, \\ the negative radial electric field is not directly related to the reduction of thermal diffusivity.
}

Previously, we reported the measurements of a radial electric field derived from a poloidal rotation velocity. ${ }^{1} \mathrm{Re}-$ cently we found that the ion temperature measured with charge exchange spectroscopy was overestimated by miscalibrating the charge coupled device (CCD) detector. Here we show the ion temperature and poloidal rotation velocity and the location of the magnetic axis for both the vacuum magnetic field and a finite $\beta$ equilibrium as arrows in Figs. 1 and 2. The main difference from previous results is the ion temperature profile for the low-density case. The Shafranov shifts estimated by using the equilibrium code VMEC were overestimated due to the lack of precise NBI deposition profiles in the previous analysis. The shift is 2.4 $\mathrm{cm}$ for the low-density case and $2.8 \mathrm{~cm}$ for the high-density case and shows good agreement with that obtained from the ion temperature profiles by taking a more accurate power deposition model.

Since the ion pressure term in the radial momentum balance equation is small, the derived electric field profiles are very similar to the previous results as shown in Fig. 3 . The measured radial electric field is still more negative

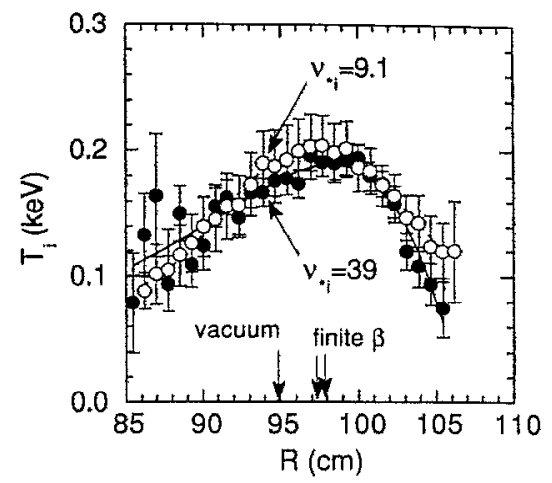

FIG. 1. Profiles of ion temperature measured with charge exchange spectroscopy, where $v_{*}$ is the normalized ion collisionality evaluated at half of the plasma minor radius. than the neoclassical prediction. More recently fast ion loss and charge exchange flux, which were not included in the previous analysis, have been taken into account. ${ }^{2}$ The ab-

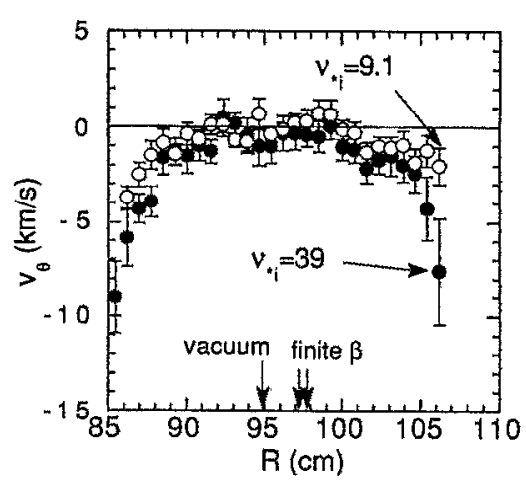

FIG. 2. Profiles of poloidal rotation velocity measured with charge exchange spectroscopy, where $v_{i}$ is the normalized ion collisionality evaluated at half of the plasma minor radius.

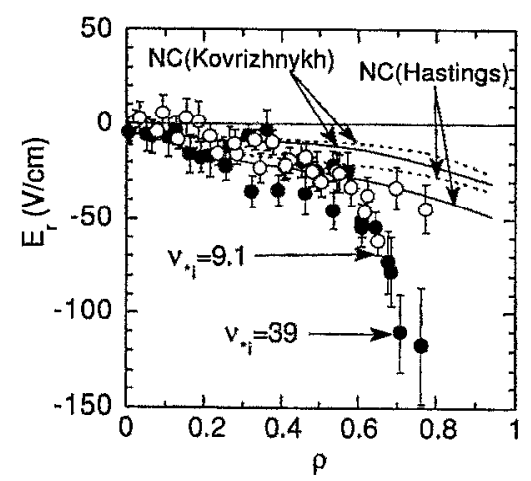

FIG. 3. Radial electric field profiles measured in CHS and neoclassical estimates. Solid lines are the calculated radial electric field for the highdensity plasma and the dashed lines are for the low-density plasma. 


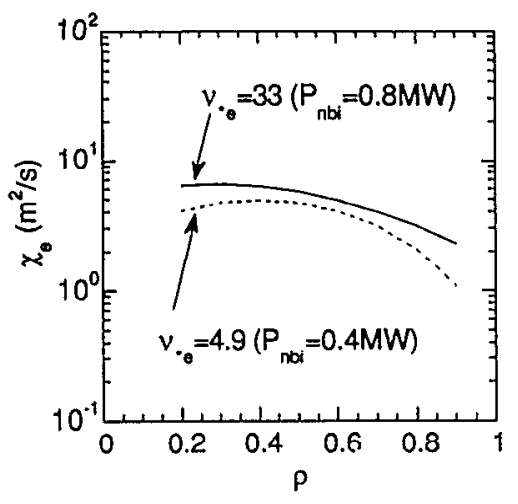

FIG. 4. Raial profiles of electron thermal diffusivity analyzed with the ORNL PROCTR-MOD code for the low- and high-density plasma.

solute value of radial electric field is still more negative than the theoretical prediction. The thermal diffusivily decreases toward the plasma edge for both low- and highdensity cases as it did previously as shown in Fig. 4. However, the thermal diffusivity seems to be not directly related to the radial electric field. Although the radial electric field is more negative, the electron thermal diffusivity is higher for higher density. This increase of thermal diffusivity is also due to the factor of 2 increase in the absorbed NBI power. The parameter dependence of electron thermal diffusivity has been studied in more detail. ${ }^{3}$ The radial profiles of radial electric field and electron thermal diffusivity imply that the reduction of thermal diffusivity is associated with a negative electric field, however, parameter dependencies of the radial electric field and diffusivity are unclear.

${ }^{1}$ K. Ida, H. Yamada, H. Iguchi, S. Hidekuma, H. Sanuki, K. Yamazaki, and the CHS Group, Phys. Fluids B 3, 515 (1991).

${ }^{2}$ H. Sanuki, K. Itoh, K. Ida, and S. I-Itoh, submitted to J. Phys. Soc. Jpn. ${ }^{3}$ H. Yamada, K. Ida, H. Iguchi, H. C. Howe, S. Kubo, Y. Ogawa, K. Hanatani, D. Okamura, H. Arimoto, M. Nosokawa, H. Idei, O. Kaneko, K. Matsuoka, S. Morita, K. Nishimura, N. Noda, T. Ozaki, A. Sagara, H. Sanuki, Y. Takeiri, Y. Takita, C. Takahashi, K. Tsuzuki, in Proceedings of 18th European Conference on Controlled Fusion and Plasma Physics, Berlin (European Physical Society, Petit-Lancy, Switzerland, 1991), Vol. 15C, Part II, p. 137. 